\title{
Isolation of Small Extracellular Vesicles From Human Serum Using a Combination of Ultracentrifugation With Polymer-Based Precipitation
}

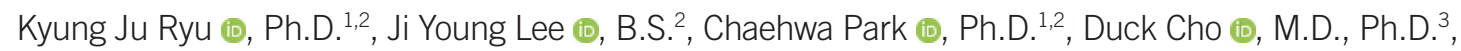 \\ and Seok Jin Kim (i), M.D., Ph.D. ${ }^{1,4}$ \\ ${ }^{1}$ Department of Health Sciences and Technology, Samsung Advanced Institute for Health Sciences and Technology, Sungkyunkwan University, Seoul, Korea; \\ ${ }^{2}$ Samsung Biomedical Research Institute, Samsung Medical Center, Seoul, Korea; ${ }^{3}$ Department of Laboratory Medicine and Genetics, Samsung Medical \\ Center, Sungkyunkwan University School of Medicine, Seoul, Korea; ${ }^{4}$ Division of Hematology and Oncology, Department of Medicine, Samsung Medical \\ Center, Sungkyunkwan University School of Medicine, Seoul, Korea
}

Methods for reproducibly isolating and enriching small extracellular vesicles (EVs) from blood are essential for clinical utilization of small EVs in cancer patients. We combined ultracentrifugation (UC) with polymer-based precipitation (ExoQuick [EQ] or Total Exosome Isolation [TEI] kit) to isolate small EVs (diameter, 30-150 nm) from the serum of breast cancer patients. We compared the performance of four cycles of UC (UC4x) with that of two cycles of $U C$ followed by enrichment using the $E Q(U C 2 x \rightarrow E Q)$ or TEl (UC2 $x \rightarrow T E I)$ kits. The mean concentration of small EVs isolated from $1 \mathrm{~mL}$ of serum using UC2x $\rightarrow E Q$ $(139.0 \pm 29.1 \mu \mathrm{g})$ and $U C 2 x \rightarrow$ TEl $(140.4 \pm 5.0 \mu \mathrm{g})$ did not differ from that obtained using UC4x $(141.8 \pm 26.9 \mu g)$. The mean number of EV particles obtained using UC4x was $29.2 \pm$ $9.9 \times 10^{9}$ per $\mathrm{mL}$ of serum, whereas $\mathrm{UC} 2 \mathrm{x} \rightarrow \mathrm{EQ}$ and $\mathrm{UC} 2 \mathrm{x} \rightarrow \mathrm{TEl}$ yielded higher numbers of EVs $\left(50.7 \pm 17.0 \times 10^{9}\right.$ and $59.3 \pm 20.6 \times 10^{9}$, respectively). Concentrations of EV microRNAs, including miR-21 and miR-155, did not differ between the three methods. In conclusion, performing UC prior to the use of polymer-based precipitation kits could be feasible for isolating small EVs from human serum in large sample-based translational researches.

Key Words: Extracellular vesicle, Precipitation, Ultracentrifugation, Isolation, Performance
Received: May 31, 2019

Revision received: September 25, 2019

Accepted: November 14, 2019

\section{Corresponding author:}

Seok Jin Kim, M.D., Ph.D.

Division of Hematology-Oncology,

Department of Medicine, Samsung Medical Center, Sungkyunkwan University School of Medicine, 81 Irwon-ro, Gangnam-gu, Seoul 06351, Korea

Tel: +82 2-3410-1766

Fax: +82 2-3410-1754

E-mail: kstwoh@skku.edu

\section{(i) (8)}

(C) Korean Society for Laboratory Medicine This is an Open Access article distributed under the terms of the Creative Commons Attribution Non-Commercial License (http://creativecommons.org/licenses/by-nc/4.0) which permits unrestricted non-commercial use, distribution, and reproduction in any medium, provided the original work is properly cited.
Extracellular vesicles (EVs), small membrane-bound structures secreted by various types of cells, are involved in cell-to-cell communication by transferring cargos such as nucleic acids [1, 2]. The delivery of nucleic acids, including microRNAs (miRNAs), through EVs to target cells may promote tumor growth and metastasis [3]. The term 'exosome' is used to describe a population of small EVs (30-150 nm) that can be discriminated by size from microvesicles (100-1,000 nm) [4, 5]. However, the Minimal Information for Studies of Extracellular Vesicles 2018 study recommended the use of 'small EVs' rather than 'exosomes' for describing EVs sized $<100 \mathrm{~nm}$ [6]. For clinical utilization of small EVs in human disorders, it is essential to reproducibly isolate and enrich small EVs from blood and body fluids [7, 8].

Currently, several methodologies exist for the isolation and analysis of small EVs including ultracentrifugation (UC), size-exclusion chromatography (SEC), immunoaffinity capture-based methods, and polymer-based precipitation kits [9-11]. Although UC has long been considered the gold standard for isolating small EVs, there is an unmet need for methods that can completely separate small EVs from other nonvesicular entities such as apoptotic bodies.

Combinations of techniques have been evaluated in a bid to 
enhance the isolation efficiency and enrichment of small EVs. Recent studies have shown that coupling one cycle of UC with SEC provided better results than UC or SEC alone [12, 13], although serum protein contamination remained a problem when using this approach [13]. However, as studies utilizing human samples for clinical purposes usually require a large number of patients, polymer-based precipitation kits are used to isolate EVs from human samples because they do not require specialized equipment and have a large and scalable sample capacity. Despite this, polymer-based precipitation has limited value because it results in larger amounts of non-EV contaminants, such as proteins and polymeric materials, than other methods [9]. To overcome this disadvantage, attempts have been made to use UC followed by enrichment using a polymer-based precipitation kit to isolate EVs from bovine milk; this combined method was reported to provide a better yield than polymer-based precipitation alone [14]. However, there are limited data on the efficiency of coupling UC with a polymer-based precipitation technique for isolating EVs from the blood of cancer patients.

We used an approach combining UC and enrichment using one of the two commercially available polymer-based precipitation kits (ExoQuick [EQ], System Biosciences, Mountain View, CA, USA; or Total Exosome Isolation [TEI], Invitrogen Life Technologies, Carlsbad, CA, USA) to compare the isolation efficiency of $U C$ alone (four cycles of UC [UC4x]) with that of UC followed by enrichment using EQ or TEI kits (two cycles of UC followed by the enrichment of small EVs using EQ [UC2x $\rightarrow E Q]$ or TEl [UC2x $\rightarrow$ TEI], respectively; Fig. 1A). The isolated small EVs were characterized according to the recommendations for $\mathrm{EV}$ research and definition [11].

Archived serum samples from breast cancer patients $(\mathrm{N}=30)$ that had been stored at $-80^{\circ} \mathrm{C}$ were used (Institutional Review Board of Samsung Medical Center, File No. SMC 2017-12-068, study period (2017-2019); the nine biosamples were provided by Samsung Medical Center BioBank, 2018-0004). As a preparation step, we first performed a brief round of low-speed centrifugation to eliminate cellular debris and large particles by sequentially centrifuging $1 \mathrm{~mL}$ of serum at 2,000 $\times g$ at $4^{\circ} \mathrm{C}$ for 10 minutes and $10,000 \times g$ at $4^{\circ} \mathrm{C}$ for 30 minutes. The supernatant was then filtered through a $0.22-\mu \mathrm{m}$ filter and ultracentrifuged at $110,000 \times g$ for 120 minutes at $4^{\circ} \mathrm{C}$.

For UC4x, the samples underwent three further cycles of UC $(110,000 \times g)$ at $4^{\circ} \mathrm{C}$ for 70 minutes, and the pellet was discarded at each step (Fig. 1A). For UC2x $\rightarrow E Q$ and $U C 2 x \rightarrow T E I$, the samples underwent two cycles of UC $(110,000 \times g)$ at $4^{\circ} \mathrm{C}$ for 120 and 70 minutes, respectively. As all tests were conducted on the same sample, each group essentially comprised three samples containing $1 \mathrm{~mL}$ of serum. The resulting pellet was reconstituted in $0.5 \mathrm{~mL}$ of phosphate-buffered saline (PBS; Gibco, NY, USA) and added to $0.1 \mathrm{~mL}$ of $E Q$ solution or TEl according to the manufacturer's protocols. The samples were incubated for $30 \mathrm{~min}$ utes at $4^{\circ} \mathrm{C}$ and centrifuged twice at $1,500 \times g$ for 30 and 5 minutes, respectively. The final pellet was reconstituted in $100 \mu \mathrm{L}$ of PBS (Fig. 1A).

The particle number of the isolated small EVs was quantified using the nanoparticle tracking analysis (NTA), and the protein concentration was assessed by the bicinchoninic acid (BCA) method using the Pierce BCA Protein Assay kit (Thermo Fisher Scientific, Rockford, IL, USA). The small EVs were characterized by immunoblotting for the presence of EV markers (Western blotting) after the isolated EVs were lysed in RIPA buffer (0.5\% sodium deoxycholate, 1\% Nonidet P-40, $150 \mathrm{mM} \mathrm{NaCl}, 50 \mathrm{mM}$ Tris [pH 7.5], $0.1 \%$ sodium dodecyl sulfate [SDS], and $1 \mathrm{mM}$ phenylmethylsulfonyl fluoride). Ten micrograms of each protein sample was electrophoresed on a 4-12\% SDS polyacrylamide gel and incubated overnight at $4^{\circ} \mathrm{C}$ with the following antibodies: anti-CD63 (sc-5275), anti-CD81 (sc-7637), anti-Alix (sc-53540), anti-TSG101 (sc-7964) (Santa Cruz Biotechnology, Santa Cruz, CA, USA), and anti-albumin (Cell signaling Technologies, Danvers, MA, USA). The morphology of the isolated small EVs was analyzed by transmission electron microscopy (TEM).

Because the nucleic acid cargo carried by small EVs is important for their potential role in cancer patients, we extracted the RNA from the small EVs to evaluate the concentration of EV cargo miRNAs. As miR-21, miR-101, miR-155, miR-223, and miR451a have been reported to be selectively enriched in EVs from patients with breast cancer, we measured their concentrations in small EVs [15]. Small EV cargo miRNA was analyzed after total RNA was extracted from the isolated small EVs using the miRNeasy Micro Kit (Qiagen, Valencia, CA, USA), according to the manufacturer's instructions. RNA concentration was measured using a NanoDrop ND-100 Spectrophotometer (NanoDrop Technologies, Wilmington, DE, USA). The RNA was reverse-transcribed using the TaqMan microRNA Reverse Transcription Kit (Thermo Fisher Scientific) reagent containing specific miRNA primers (Thermo Fisher Scientific) for five miRNAs (miR-21-5p, miR-101-3p, miR-155-5p, miR-223-3p, and miR451a), and real-time PCR (ABI PRISM 7900HT, Applied Biosystems, Foster City, USA) was performed in triplicate using the cDNA from each sample with $2 \times$ TaqMan Universal Master Mix II (with no AmpErase Uracil N-Glycosylase, UNG) and a 20x TaqMan miRNA expression assay (Thermo Fisher Scientific). 


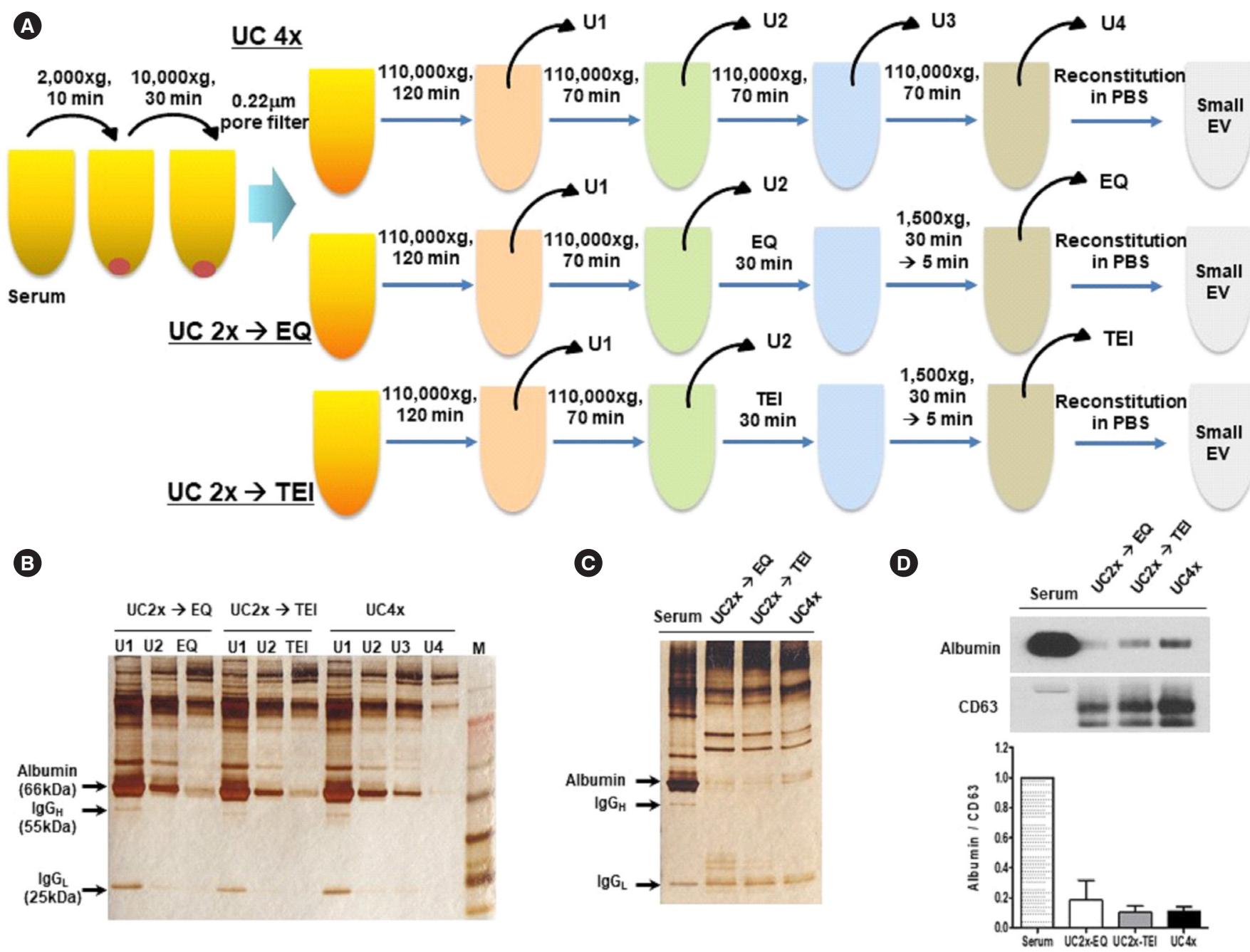

Fig. 1. Comparison of EV isolation methods. (A) Study overview: three methods for isolating EVs from serum samples of breast cancer patients. (B) Silver-stained polyacrylamide gel of supernatants from the first to the final EV enrichment steps. (C) Silver-stained polyacrylamide gel of small EVs enriched after the final step. (D) Western blotting analysis for CD63 and albumin in serum and small EVs isolated using the three methods (UC4x: four cycles of UC; UC2x $\rightarrow E Q$ : two cycles of UC followed by enrichment using the EQ kit; UC2x $\rightarrow T E I:$ two cycles of UC followed by enrichment using the TEl kit). Ten micrograms of protein were used from each sample.

Abbreviations: EV, extracellular vesicle; UC, ultracentrifugation; EQ, ExoQuick; TEI, Total Exosome Isolation kit; PBS, phosphate-buffered saline; IgGH, immunoglobulin G heavy chain; IgGL, immunoglobulin G light chain.

The differences between the three methods were analyzed by ANOVA using SPSS (version 23.0; IBM SPSS Inc., Armonk, NY, USA). $P<0.05$ was considered significant, and two-sided tests were used in all calculations. Graphs were plotted using GraphPad Prism 5.0 (GraphPad Software, Inc., San Diego, CA, USA).

Analysis of supernatants after the first round of $U C$ revealed protein bands, including albumin and immunoglobulin heavy and light chains, which diminished after the final step of each method (EQ, TEI, and U4, Fig. 1B). Silver-stained gels showed that the final pellets resuspended in PBS after the final step of the three methods (UC2x $\rightarrow E Q$, UC2 $\rightarrow T E I$, and UC4x) contained extremely low concentrations of albumin compared with the serum sample, although several bands reflecting contaminating proteins were still observed (Fig. 1C). Western blotting for albumin and CD63 using $10 \mu \mathrm{g}$ of protein from each sample also showed that the ratio of albumin to CD63 was significantly lower in the end-product of the three methods than in the serum sample (Fig. 1D). These findings indicate that UC coupled to EQ or TEl might achieve an enrichment of small EVs comparable to that obtained using UC alone, as non-EV-related proteins, such as albumin and immunoglobulins, could be eliminated. The mean concentration of small EVs isolated from $1 \mathrm{~mL}$ of serum by UC2x 

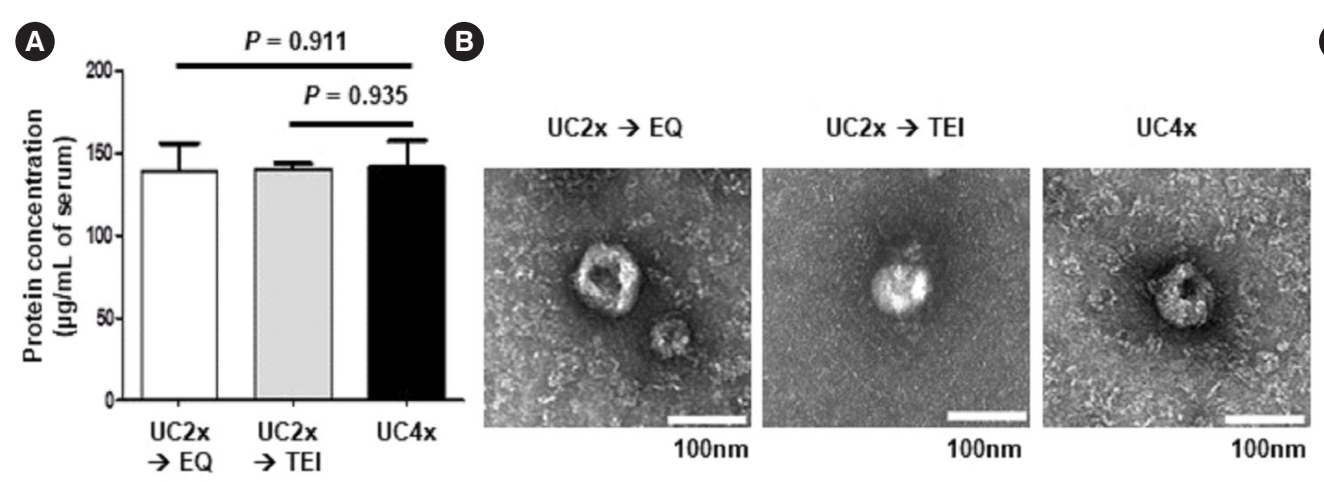

C
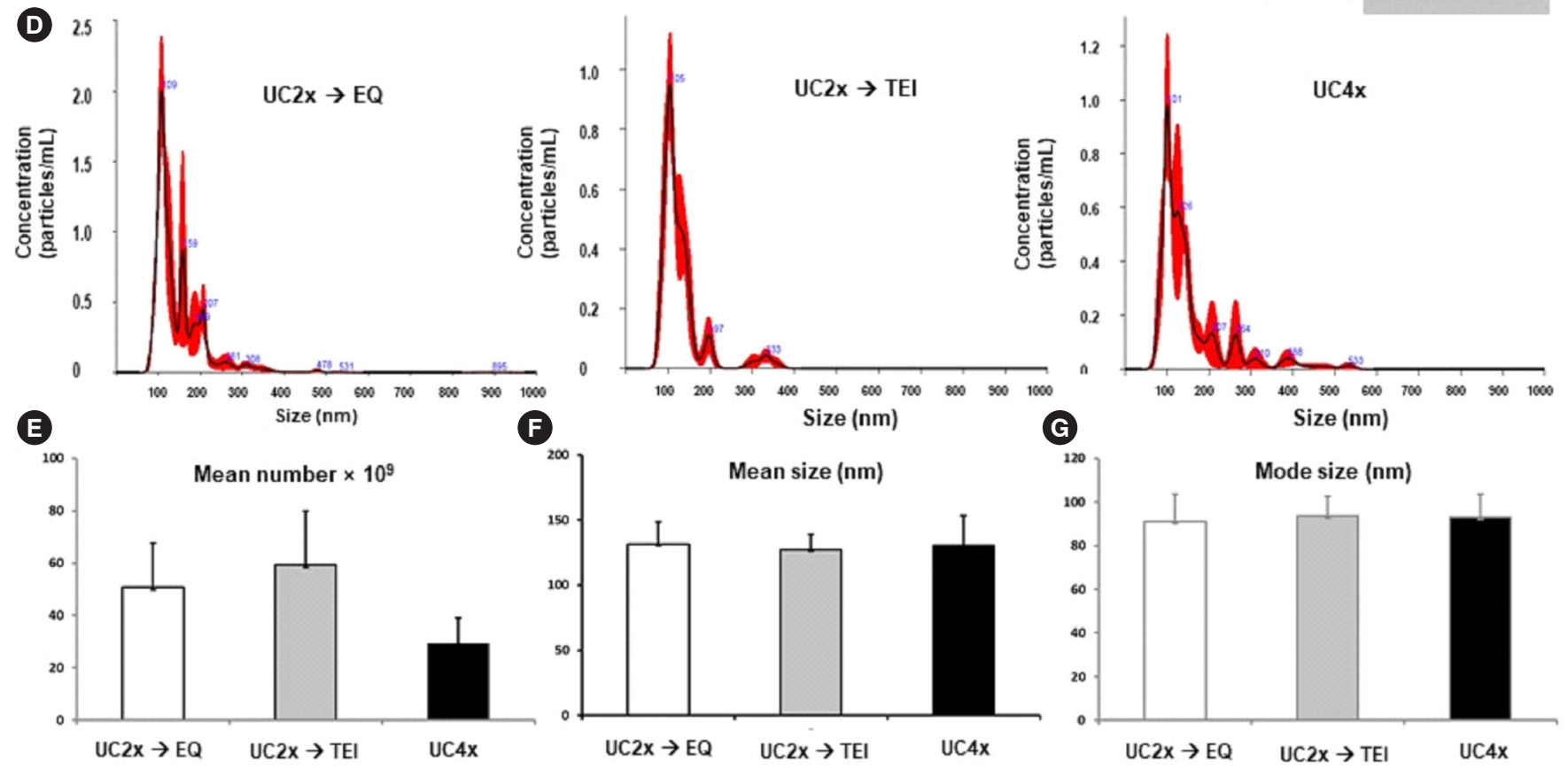

Fig. 2. Characterization of extracellular vesicle. (A) Protein concentrations in small EVs isolated using the three methods. (B) Transmission electron microscopy images of small EVs; size bar=100 nm. (C) Western blotting analysis for Alix, CD63, TSG101, and CD81 using $10 \mu \mathrm{g}$ of small EVs isolated using the three methods (UC4x: four cycles of UC; UC2x $\rightarrow E Q$ : two cycles of UC followed by enrichment using the EQ kit; UC2 $\rightarrow$ TEl: two cycles of UC followed by enrichment using the TEI kit). (D) Size distribution of small EVs and comparison of three methods using nanoparticle tracking analysis. (E-G) Comparison of mean number of EV particles, mean size, and mode size among the three methods. The data represent the mean \pm SD of three independent experiments.

Abbreviations: see Fig. 1.

$\rightarrow$ EQ and UC2x $\rightarrow$ TEl $(139.0 \pm 29.1 \mu \mathrm{g}$ and $140.4 \pm 5.0 \mu \mathrm{g}$, respectively) was not significantly different from that obtained using UC4x (141.8 $\pm 26.9 \mu \mathrm{g}$, Fig. 2A).

The concentration of small EVs we obtained was higher than that normally observed in other samples. This could be associated with the type of underlying disorders, as the concentration of small EVs isolated using UC4x from $1 \mathrm{~mL}$ of serum from healthy people and lymphoma patients in our previous pilot experiments was approximately $30 \mu \mathrm{g}$ and $70 \mu \mathrm{g}$, respectively (data not shown).

TEM imaging analysis showed that the small EVs isolated us- ing the three methods were all rounded or cup-shaped and within the expected size range (Fig. 2B). The small EVs isolated using the three methods were then characterized by Western blotting for CD63, CD81, Alix, and TSG101. Although CD63 expression was slightly lower in the small EVs isolated using $U C 2 x \rightarrow E Q$, the expression of the other EV markers, including Alix, CD81, and TSG101, did not differ significantly between the three methods (Fig. 2C).

The number and size of small EVs isolated by the three methods were analyzed using a Nanosight NS300 instrument (Malvern Instruments, Worcestershire, UK) with NTA (Fig. 2D). The 

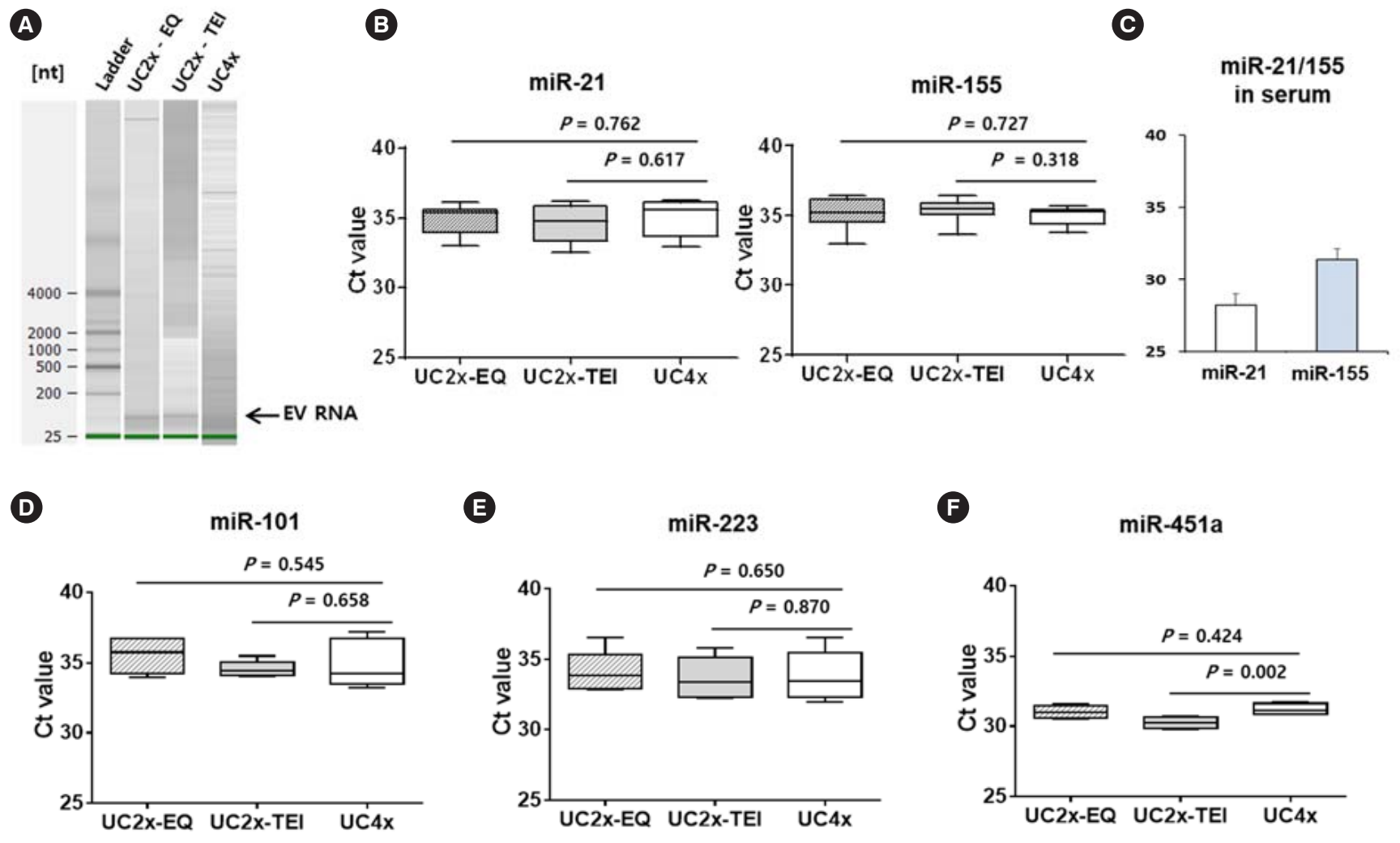

Fig. 3. Analysis of miRNA cargo in EV. (A) The quality of EV RNA was evaluated using an Agilent2100 bioanalyzer with an RNA 6000 Pico kit. (B) miR-21 and miR-155 concentrations in the small EVs did not differ among the three methods. (C) Serum concentrations of miR-21 and miR-155. (D-F) miR-101, miR-223, and miR-451a concentrations did not differ among the three methods. The data represent the mean \pm SD of three independent experiments. Boxes and whiskers indicate the minimum-maximum, and the lines represent the median of three independent experiments ( $B, D, E$, and $F)$.

Abbreviations: miRNA, microRNA; EV, extracellular vesicle; UC, ultracentrifugation; EQ, ExoQuick; TEI, Total Exosome Isolation kit; UC4x, four cycles of UC; UC2x-EQ, two cycles of UC followed by enrichment using the EQ kit; UC2x-TEI, two cycles of UC followed by enrichment using the TEI kit.

mean number of EV particles was $29.2 \pm 9.9 \times 10^{9}$ per $\mathrm{mL}$ of serum for UC4x, whereas $U C 2 x \rightarrow E Q$ and $U C 2 x \rightarrow T E I$ yielded a higher number of $\mathrm{EV}$ particles; however, there was no significant difference between $\mathrm{UC} 2 \mathrm{x} \rightarrow \mathrm{EQ}\left(50.7 \pm 17.0 \times 10^{9}\right.$ per $\mathrm{mL}$ of serum) and UC2x $\rightarrow$ TEI $\left(59.3 \pm 20.6 \times 10^{9}\right.$ per $\mathrm{mL}$ of serum, Fig. 2E). The mean diameters (UC2x $\rightarrow E Q: 131.3 \pm 17.4 \mathrm{~nm}, \mathrm{UC} 2 \mathrm{x} \rightarrow$ TEl: $127.4 \pm 11.8 \mathrm{~nm}$, and UC4x: $130.5 \pm 23.1 \mathrm{~nm}$ ) and mode diameters of the small EVs isolated using the three methods did not differ significantly (Fig. 2F, G).

Taken together, these results indicate that $1 \mathrm{~mL}$ of serum could be the minimum volume required for our new method, similar to the UC method. As we had no internal control for EV miRNA, we extracted RNA from equal amounts of serum and determined miRNA concentrations. The concentrations of miR-21 and miR155 in the small EVs isolated did not differ significantly among the three methods (Fig. 3A, B); however, their concentrations differed from those in serum samples (Fig. $3 \mathrm{C}$ ). The difference between serum concentrations of miRNA and EV cargo miRNA might be associated with the different roles of circulating free miRNAs and exosomal miRNAs. The concentrations of miR-101, miR-223, and miR-451a also did not differ among the three methods (Fig. 3D-F).

In conclusion, our results suggest that performing $\mathrm{UC}$ prior to using a polymer-based precipitation kit could be a feasible and convenient method for isolating small EVs from human serum in terms of yield and quality of EV cargos such as miRNAs. This approach may be useful for large sample-based translational researches, and further studies are warranted to validate its reliability for clinical application.

\section{DATA AVAILABILITY}

Please contact the corresponding author for access to the underlying data. 


\section{ACKNOWLEDGEMENTS}

We thank all our colleagues for participating in the research and express our gratitude to the 20-20 project of Samsung Medical Center and the National Research Foundation of Korea (NRF2017R1A2B4005136).

\section{AUTHOR CONTRIBUTIONS}

Conception and design: SJK. Acquisition of data and experiments: KJR, JYL. Analysis and interpretation of data: KJR, CP, DC, SJK. Drafting of manuscript: KJR, SJK. All authors read and approved the final manuscript.

\section{CONFLICTS OF INTEREST}

No potential conflicts of interest relevant to this article are reported.

\section{RESEARCH FUNDING}

This study was supported by a grant from the Basic Science Research Program through the National Research Foundation of Korea (NRF), which is funded by the Ministry of Education, Science, and Technology (NRF-2017R1A2B4005136).

\section{ORCID}

\section{Kyung Ju Ryu Ji Young Lee Chaehwa Park Duck Cho}

Seok Jin Kim https://orcid.org/0000-0003-0986-1475 https://orcid.org/0000-0003-4396-5091 https://orcid.org/0000-0001-7624-304X https://orcid.org/0000-0001-6861-3282 https://orcid.org/0000-0002-2776-4401

\section{REFERENCES}

1. Sharma A, Khatun Z, Shiras A. Tumor exosomes: cellular postmen of cancer diagnosis and personalized therapy. Nanomedicine (Lond) 2016; 11:421-37.

2. EL Andaloussi S, Mäger I, Breakefield XO, Wood MJ. Extracellular vesicles: biology and emerging therapeutic opportunities. Nat Rev Drug Discov 2013;12:347-57.

3. Tkach $M$ and Théry $C$. Communication by extracellular vesicles: where we are and where we need to go. Cell 2016;164:1226-32.

4. Cocucci E and Meldolesi J. Ectosomes and exosomes: shedding the confusion between extracellular vesicles. Trends Cell Biol 2015;25:36472 .

5. Raposo G and Stoorvogel W. Extracellular vesicles: exosomes, microvesicles, and friends. J Cell Biol 2013;200:373-83.

6. Théry C, Witwer KW, Aikawa E, Alcaraz MJ, Anderson JD, Andriantsitohaina R, et al. Minimal information for studies of extracellular vesicles 2018 (MISEV2018): a position statement of the International Society for Extracellular Vesicles and update of the MISEV2014 guidelines. J Extracell Vesicles 2018;7:1535750.

7. Skog J, Würdinger T, van Rijn S, Meijer DH, Gainche L, Sena-Esteves M, et al. Glioblastoma microvesicles transport RNA and proteins that promote tumour growth and provide diagnostic biomarkers. Nat Cell Biol 2008; 10:1470-6.

8. Melo SA, Luecke LB, Kahlert C, Fernandez AF, Gammon ST, Kaye J, et al. Glypican-1 identifies cancer exosomes and detects early pancreatic cancer. Nature 2015;523:177-82.

9. Li P, Kaslan M, Lee SH, Yao J, Gao Z. Progress in exosome isolation techniques. Theranostics 2017;7:789-804.

10. Lobb RJ, Becker M, Wen SW, Wong CS, Wiegmans AP, Leimgruber A, et al. Optimized exosome isolation protocol for cell culture supernatant and human plasma. J Extracell Vesicles 2015;4:27031.

11. Lötvall J, Hill AF, Hochberg F, Buzás El, Di Vizio D, Gardiner C, et al. Minimal experimental requirements for definition of extracellular vesicles and their functions: a position statement from the International Society for Extracellular Vesicles. J Extracell Vesicles 2014;3:26913.

12. Koh YQ, Almughlliq FB, Vaswani K, Peiris HN, Mitchell MD. Exosome enrichment by ultracentrifugation and size exclusion chromatography. Front Biosci (Landmark Ed) 2018;23:865-74.

13. An M, Wu J, Zhu J, Lubman DM. Comparison of an optimized ultracentrifugation method versus size-exclusion chromatography for isolation of exosomes from human serum. J Proteome Res 2018;17:3599-605.

14. Yamada T, Inoshima Y, Matsuda T, Ishiguro N. Comparison of methods for isolating exosomes from bovine milk. J Vet Med Sci 2012;74:1523-5.

15. Melo SA, Sugimoto H, O'Connell JT, Kato N, Villanueva A, Vidal A, et al. Cancer exosomes perform cell-independent microRNA biogenesis and promote tumorigenesis. Cancer Cell 2014;26:707-21. 